\title{
Determinants of delayed gastric emptying in anorexia nervosa and bulimia nervosa
}

\author{
P H ROBINSON, M CLARKE, AND J BARRETT \\ From the Dept of Psychiatry, Institute of Psychiatry, London and Dept of Nuclear Medicine and Dept of \\ Medical Physics, King's College Hospital, Denmark Hill, London
}

SUMMARY Gastric emptying was measured using a gamma camera in 22 patients with anorexia nervosa, in 10 patients of normal or high weight with bulimia nervosa and in 10 controls. Patients with anorexia nervosa were tested (1) while underweight and selecting their own diet (10 patients); (2) underweight, but receiving an adequate diet on an inpatient unit (refeeding diet) (12 patients); and (3) under refeeding diet conditions after weight gain (eight patients). Three meals, each labelled with technetium $99 \mathrm{~m}$-sulphur colloid, $3 \cdot 7 \mathrm{MBq}$ were used: (1) a mixed solid meal containing labelled poached egg; (2) $200 \mathrm{ml}$ d-glucose solution, $0.5 \mathrm{kcal} / \mathrm{ml}$, and (3) $200 \mathrm{ml}$ physiological saline. Only gastric emptying rates of the solid meal and glucose solution were significantly delayed. Gastric emptying of saline was normal. The gastric disturbance was confined to patients with anorexia nervosa selecting their own diet. Patients receiving adequate nutrition on the ward had normal gastric emptying and weight gain in this group had no significant effect on emptying. Slow emptying was observed in patients who maintained a low weight solely by food restriction as well as in patients whose anorexia nervosa was complicated by episodes of bulimia. Thus, slow gastric emptying occurred when the quantity of food reaching the duodenum was sufficiently reduced to result in severe weight loss. Moreover, abnormal gastric emptying was seen only after the two meals that contained calories and were hypertonic to plasma, either of which properties could mediate the disturbance. Gastric emptying in bulimia nervosa was normal. Slow gastric emptying could exacerbate undereating in starving patients with anorexia nervosa by enhancing satiety.

Gastric distension is a potent inhibitor of feeding, and the demonstration of delayed gastric emptying in patients with anorexia nervosa ${ }^{1-5}$ suggests that gastric retention might contribute to the maintenance of undereating in this condition. The mechanisms underlying delayed emptying, and whether it is a primary disorder or secondary to other disturbances are, however, unknown and we have investigated gastric emptying of various meals in anorexic patients at different stages of nutritional treatment in an attempt to identify the determinants of delayed gastric emptying in anorexia nervosa. In the closely related condition, bulimia nervosa, ${ }^{6}$ patients succumb to episodes of massive overeating, suggesting a failure of satiety. Because an acceleration of gastric emptying has been proposed as a factor mediating overeating in morbid obesity ${ }^{7}$ we studied

\footnotetext{
Address for correspondence: Dr P H Robinson, Academic Dept of Psychological Medicine, King's College Hospital School of Medicine and Dentistry, Denmark Hill, London SE5 9RS.

Received for publication 24 September 1987.
}

gastric function in a group of patients with bulimia nervosa in order to elucidate the role of disturbed gastric emptying in the pathogenesis of this condition.

\section{Methods}

\section{SUBJECTS}

\section{Anorexia nervosa}

Twenty two patients (21 women, one man), with anorexia nervosa were studied. They fulfilled the diagnostic criteria of Russell, ${ }^{8}$ all having self-imposed severe weight loss, amenorrhoea of at least six months duration and a morbid fear of fatness. Ten patients were investigated while selecting their own diet either as outpatients (seven patients) or as inpatients undergoing observation without refeeding (three patients). Twelve patients with anorexia nervosa were tested between one and four weeks after admission to hospital (mean 14 days \pm 5.77 $(\mathrm{SD}))$ while underweight but receiving a mixed diet 
of 2500-3000 Kcal per day. Eight patients were retested when they had regained weight on the ward, while still undergoing inpatient refeeding. Testing of patients was therefore carried out under three distinct conditions: (1) Underweight, but selecting their own diet. (2) Underweight, but receiving the refeeding diet. (3) Weight restored, and receiving the refeeding diet.

Among the 22 patients with anorexia nervosa, 10 suffered from episodes of bulimia associated with vomiting, abuse of laxatives or prolonged starvation. Of the 12 patients tested while under supervision and receiving the refeeding diet none admitted to bulimic symptoms in the two to three weeks they had been in hospital, although eight patients had experienced persistent bulimia up to the time of admission. Of the 10 patients tested while selecting their own diet, four had episodes of bulimia at least weekly and all four reported at least one episode in the week before testing.

\section{Bulimia nervosa}

Ten female outpatients who were not currently underweight and who fulfilled criteria ${ }^{6}$ for bulimia nervosa were tested. They all suffered episodes of massive overeating at a frequency of at least one episode per week. Eight of these patients, all within $15 \%$ of mean population matched weight, ${ }^{,}$practised self-induced vomiting after episodes of bulimia, and of these, five regularly took large doses of laxatives. The other two patients, weighing more than $115 \%$ of MPMW (123\% and $170 \%$ MPMW), used selfstarvation to compensate for calories ingested during bulimic episodes. All these patients showed a morbid fear of fatness similar to that observed in the patients with anorexia nervosa.

\section{Controls}

Ten normal control subjects in the same age range as the patients were recruited, eight women and two men. All were within $15 \%$ of mean population matched weight ${ }^{\prime}$ and denied symptoms of eating disorder and of other psychiatric illness. Their scores on the Eating Attitudes Test ${ }^{11}$ and on the Dutch Eating Behaviour Questionnaire" were within reported normal limits.

Clinical and demographic data on patients and controls are given in Table 1.

Patients and controls were asked to refrain from taking any medication, apart from benzodiazepine hypnotics, for at least one week before testing, and to fast and avoid smoking from midnight on the night before each test. Subjects were also asked to drink a large glass of water before bed on the night before a test. This was found to encourage gastric emptying of solid particles of food which otherwise reduced the quality of results after ingestion of labelled liquid meals because of adsorption of radioactive label onto the solid.

GASTRIC EMPTYING TESTS

All tests were done using an IGE Maxicamera gamma camera and the Adac image analysis system. Caloric and acaloric liquid meals empty differently from each other, ${ }^{12}$ and liquid meals show different emptying patterns compared with solids. ${ }^{13}$ The emptying of each of these three types of meal was therefore examined separately. A mixed solid meal, a glucose solution, and a saline solution were used and each series of three studies was completed within a week. Ethical approval and informed consent were obtained.

SOLID MEAL

Sixty millilitres of egg white were mixed with 3.7 MBq (100 microcuries) of Technetium $99 \mathrm{~m}$-sulphur colloid and poached for 10 minutes in a domestic poacher. The egg was then sliced in two directions at right angles using an egg slicer, resulting in roughly

Table 1 Demographic and clinical data

\begin{tabular}{|c|c|c|c|c|}
\hline \multirow[b]{3}{*}{ Controls } & \multirow{3}{*}{$\begin{array}{l}\text { Bulimia } \\
\text { nervosa }\end{array}$} & \multicolumn{3}{|c|}{ Anorexia nervosa } \\
\hline & & \multicolumn{2}{|c|}{ Inpatient refeeding } & \multirow{2}{*}{$\begin{array}{l}\text {-Self-selection } \\
\text { diet }\end{array}$} \\
\hline & & Low wt. & Wt. rest & \\
\hline \multicolumn{5}{|l|}{ Age } \\
\hline $28 \cdot 7$ & $25 \cdot 1$ & $26 \cdot 5$ & $23 \cdot 4$ & $32 \cdot 2$ \\
\hline SD 4.42 & $5 \cdot(02$ & $7 \cdot 5$ & $4 \cdot 12$ & $15 \cdot 24$ \\
\hline n $\quad 10$ & 10 & 12 & 8 & 10 \\
\hline \multicolumn{5}{|c|}{ Length of history (years) } \\
\hline 0 & $8 \cdot 1$ & $5 \cdot 9$ & $6 \cdot 0$ & 9.44 \\
\hline SD & $4 \cdot 63$ & $4 \cdot 4$ & $4 \cdot 83$ & $11 \cdot 67$ \\
\hline \multicolumn{5}{|c|}{ Body mass index (kg/sq metre) } \\
\hline $21 \cdot 87^{a}$ & $23 \cdot 68^{a}$ & $14 \cdot 70^{\mathrm{h}}$ & $18 \cdot 63$ & $16 \cdot 29^{b}$ \\
\hline SD $1 \cdot 50$ & $4 \cdot 88$ & $1 \cdot 44$ & $1 \cdot 69$ & $2 \cdot 35$ \\
\hline \multicolumn{5}{|l|}{ Weight (kg) } \\
\hline $61 \cdot 67^{a}$ & $66 \cdot 62^{a}$ & $40 \cdot 8^{\mathrm{b}}$ & $51 \cdot 60$ & $45 \cdot 34^{b}$ \\
\hline SD 7.9 & $18 \cdot 21$ & $5 \cdot 2$ & $7 \cdot 07$ & $5 \cdot 95$ \\
\hline \multicolumn{5}{|c|}{ Lowest weight ever $(\mathrm{kg})$} \\
\hline $54 \cdot 22^{a}$ & $52 \cdot 47^{a}$ & $32 \cdot 61^{\mathrm{h}}$ & & $36 \cdot 33^{\mathrm{b}}$ \\
\hline SD $9 \cdot 45$ & $7 \cdot 48$ & $4 \cdot 69$ & & $5 \cdot 85$ \\
\hline \multicolumn{5}{|c|}{ Highest weight ever (kg) } \\
\hline $66 \cdot 9$ & 73.9 & $55 \cdot 32$ & & $60 \cdot 41$ \\
\hline SD $8 \cdot() 8$ & $19 \cdot 34$ & $7 \cdot 17$ & & $9 \cdot 62$ \\
\hline \multicolumn{5}{|c|}{ Bulimic episodes (number (\%) of subjects) } \\
\hline 0 & $10(100)$ & $6(50)$ & $4(50)$ & $4(40)$ \\
\hline \multicolumn{5}{|c|}{ Self-induced vomiting (number (\%) of subjects) } \\
\hline 0 & $8(80)$ & $6(50)$ & $4(50)$ & $3(30)$ \\
\hline \multicolumn{5}{|c|}{ Laxative abuse (number ( $\%$ ) of subjects) } \\
\hline 0 & $4(40)$ & $2(16 \cdot 7)$ & $2(25)$ & $2(20)$ \\
\hline
\end{tabular}

Means with different superscripts are significantly different $(\mathrm{p}<0 \cdot 05)$ in an ANOVA including: controls, bulimia nervosa, anorexia nervosa (refeeding diet, low weight), and anorexia nervosa (self-selection diet). 
cuboidal particles of average volume $0.366 \mathrm{ml}$. This was served hot with $150 \mathrm{ml}$ fresh orange juice and one slice of medium cut white bread and $2.5 \mathrm{~g}$ butter. The total caloric value of the meal, determined from tables, was $155 \mathrm{Kcal}$, its volume, after liquidising in a domestic blender, was $235 \mathrm{ml}$ and the mean caloric concentration was therefore $1.52 \mathrm{Kcal} / \mathrm{ml}$. An aliquot of the liquidised solid meal was centrifuged twice at $5000 \mathrm{rpm}$ for five minutes and the supernatant analysed using the ion selective electrode technique for electrolytes, depression of freezing point for osmolality, and glucose oxidase. This revealed the following constituents: sodium $68 \mathrm{mmol} / \mathrm{l}$, potassium $42 \mathrm{mmol} / \mathrm{l}$, glucose $97 \mathrm{mmol} / \mathrm{l}$, and osmotic pressure $569 \mathrm{mosmol} / \mathrm{l}$ (about $2 \times$ plasma tonicity). After in vitro incubation for two hours in $0 \cdot 1 \mathrm{~N}$ hydrochloric acid a sample of labelled egg retained $90 \cdot 74 \%$ of the original radioactivity, allowing for decay, showing that the label would remain bound to the food particles in the acid environment of the stomach.

The meal was served to the subject and the time taken to complete it noted. Imaging began as soon as the meal ended. A 60 second static anterior scan followed immediately by a 60 second posterior scan were recorded every few minutes in the erect posture until two hours after the beginning of the meal. The two views were taken to compensate for anteroposterior movement of the meal within the stomach. ${ }^{14}$ Each image was displayed on the computer screen and a region of interest drawn electronically around the gastric outline. The geometric means of anterior and posterior gastric counts were corrected for decay and a time activity curve generated.

\section{LIQUID MEALS}

Two liquid meals were used in each study $-200 \mathrm{ml} \mathrm{d}$ glucose, $0.5 \mathrm{Kcal} / \mathrm{ml}(125 \mathrm{~g} / \mathrm{l})$, and $200 \mathrm{ml}$ of physiological saline, $9 \mathrm{~g} / \mathrm{l}$, both at room temperature and both labelled with Tc99m-sulphur colloid, 3.7 MBq. After ingestion of the meal imaging was carried out using continuous, dynamic, anterior acquisition for 60 minutes with the subject lying on a couch, propped up at $60^{\circ}$ to the horizontal. Images were acquired at 15 second intervals and counts within the gastric region of interest for the 240 images were corrected for decay and plotted against time.

\section{STATISTICAL ANALYSIS}

The four groups of subjects (anorexia nervosa at low weight (self-selection diet), anorexia nervosa at low weight (refeeding diet), bulimia nervosa, and controls) were compared with each other using one way analysis of variance. No subject appeared more than once in these analyses. Four patients were tested under inpatient refeeding and self-selection diet conditions. Their results were included only in the latter group. For each analysis, the overall F-test result is given and if appropriate, post-hoc analysis was carried out using Duncan's test. The influence of differences between subjects - for example, the presence of bulimia, self-induced vomiting or laxative abuse, was determined using multivariate analysis of variance. The effect of weight gain was studied using paired $t$-tests. In all comparisons differences were taken as significant if $p=$ or $<0 \cdot 05$. One anorexic patient on the refeeding diet had the solid meal but refused to take the liquid meals.

\section{Results}

SUBJECT CHARACTERISTICS (Table 1)

One way analysis of variance revealed no significant

Table 2 Results of gastric emptying studies

\begin{tabular}{|c|c|c|c|c|}
\hline \multirow[b]{3}{*}{ Controls } & \multirow[b]{3}{*}{$\begin{array}{l}\text { Bulimia } \\
\text { nervosa }\end{array}$} & \multicolumn{3}{|c|}{ Anorexia nervosa } \\
\hline & & \multicolumn{3}{|c|}{ Inpatient refeeding } \\
\hline & & Low wt. & Wt. rest & $\begin{array}{l}\text { Self-selection } \\
\text { diet }\end{array}$ \\
\hline \multicolumn{5}{|l|}{ Solid meal } \\
\hline \multicolumn{5}{|c|}{ Duration of meal (minutes): } \\
\hline $4 \cdot 35^{\mathrm{a}}$ & $5 \cdot 16^{\mathrm{a}}$ & $6 \cdot 95^{\mathrm{b}}$ & $5 \cdot 50$ & $7 \cdot 88^{b}$ \\
\hline SD $1 \cdot 36$ & $1 \cdot 56$ & $2 \cdot 55$ & $1 \cdot 31$ & $3 \cdot 15$ \\
\hline n 10 & 10 & 12 & 8 & 10 \\
\hline \multicolumn{5}{|c|}{ Lag phase (minutes): } \\
\hline 22.95 & $23 \cdot 8$ & $19 \cdot 3$ & $26 \cdot 13$ & $31 \cdot 3$ \\
\hline SD $10 \cdot 15$ & $5 \cdot 47$ & $9 \cdot 5$ & $5 \cdot 54$ & $17 \cdot 9$ \\
\hline \multicolumn{5}{|c|}{$\%$ remaining at 100 minutes: } \\
\hline $21 \cdot 1^{a}$ & $22 \cdot 59^{\mathrm{a}}$ & $30 \cdot 0^{\mathrm{a}}$ & $28 \cdot 38$ & $45 \cdot 84^{\mathrm{b}}$ \\
\hline SD 14.42 & $14 \cdot 5$ & $18 \cdot 1$ & $18 \cdot 16$ & $20 \cdot 38$ \\
\hline \multicolumn{5}{|c|}{ Glucose meal } \\
\hline \multicolumn{5}{|c|}{ Duration of meal (minutes): } \\
\hline 0.898 & 0.958 & $1 \cdot 28$ & $0 \cdot 83$ & $1 \cdot 85$ \\
\hline SD $0 \cdot 20$ & 0.47 & $0 \cdot 88$ & 0.42 & 1.08 \\
\hline \multicolumn{5}{|c|}{ Lag phase (minutes): } \\
\hline $2 \cdot 4$ & $2 \cdot 42$ & $3 \cdot 81$ & 3.92 & $3 \cdot 0$ \\
\hline SD 1.89 & 1.80 & $3 \cdot 13$ & $2 \cdot 84$ & $1 \cdot 88$ \\
\hline \multicolumn{5}{|c|}{$\%$ remaining at 30 minutes: } \\
\hline $73 \cdot 88^{\mathrm{a}}$ & $72 \cdot 86^{a}$ & $73 \cdot 7^{\mathrm{a}}$ & $77 \cdot 25$ & $87 \cdot 20^{\mathrm{b}}$ \\
\hline SD 11.97 & $13 \cdot 40$ & $13 \cdot 6$ & $6 \cdot 61$ & $9 \cdot 46$ \\
\hline \multicolumn{5}{|l|}{ Saline meal } \\
\hline \multicolumn{5}{|c|}{ Duration of meal (minutes): } \\
\hline 1.05 & 1.87 & 1.91 & $1 \cdot 01$ & $3 \cdot 35$ \\
\hline SD 0.498 & $1 \cdot 21$ & $1 \cdot 5$ & 0.435 & $4 \cdot 57$ \\
\hline \multicolumn{5}{|c|}{ Lag phase (minutes): } \\
\hline $2 \cdot 01$ & $3 \cdot 25$ & $4 \cdot 6$ & $4 \cdot 36$ & $3 \cdot 31$ \\
\hline SD 1.55 & $2 \cdot 33$ & 5.75 & 6.05 & $1 \cdot 80$ \\
\hline \multicolumn{5}{|c|}{$\%$ remaining at 30 minutes: } \\
\hline $37 \cdot 25$ & $39 \cdot 24$ & $46 \cdot 6$ & $57 \cdot 74$ & $48 \cdot 15$ \\
\hline $\mathrm{SD} 26 \cdot 16$ & $24 \cdot 39$ & $19 \cdot 5$ & 27.06 & $28 \cdot 34$ \\
\hline
\end{tabular}

Means with different superscripts are significantly different in an ANOVA including: controls, bulimia nervosa, anorexia nervosa (refeeding diet, low weight), and anorexia nervosa (self-selection diet). 
difference between the groups in age. Body mass index $\left(\mathrm{kg} / \mathrm{metre}^{2}\right)$ was significantly lower in both groups of patients with anorexia nervosa compared with controls and patients with bulimia nervosa $(F(3,41)=24 \cdot 6, p<0 \cdot 0001)$. After inpatient treatment, lasting an average of 62 days, anorexic patients gained a mean of $10.33 \mathrm{~kg}$ in weight, a significant increase (Paired $t$-test: $\mathrm{t}=8 \cdot 28, \mathrm{df}=6, \mathrm{p}<0 \cdot 001$ ).

MEAL DURATION (Table 2)

Control subjects completed the solid meal in a mean time of 4.35 minutes. Low weight patients on the selfselection and the refeeding diets took significantly longer to finish the solid meal than controls (one way analysis of variance: $F(3,41)=4 \cdot 68, p=0 \cdot 007)$. In contrast, there was no significant difference in the time taken to finish the liquid meals, although selfselecting anorexic patients tended to consume the glucose at a slower rate than controls $(F(3,36)=2 \cdot 6$, $\mathrm{p}<0.07)$. Comparing anorexic patients before and after weight gain showed no significant change in the time taken to complete any meal (paired $t$-tests, $p>0 \cdot 1)$. Glucose was consumed faster than saline (repeated measures ANOVA; within-subject differences in liquid meal duration: $F(1,35)=7 \cdot 65$, $\mathrm{p}=0 \cdot 009$ ).

GASTRIC EMPTYING (Table 2)

The emptying of all three meals was preceded by a lag phase during which no gastric emptying was seen. The length of this phase was determined by noting the first scan on which activity was observed in the duodenum.

In several of the studies, there was some overlap between jejunal and gastric counts which introduced a degree of error into the analyses. To allow for this, we checked our results by using only frames within a study which did not show overlap and ascertaining that results from those frames were consistent with those from the whole study.

The shape of gastric emptying curves of the three meals varied considerably between subjects. Gastric emptying curves have been described as following various shapes of curve including, commonly, linear and exponential functions, ${ }^{12}{ }^{15}$ and our data were analysed to establish which of these two functions best described each curve. Linear regression was undertaken using untransformed and log transformed gastric counts. The end of the lag period was used in the regression as the beginning of the curve. Each curve was assigned to either a linear or an exponential function depending on which regression line yielded the best fit (lowest mean squared residuals). For the solid meal, $35.3 \%$ emptied linearly and $64.7 \%$ according to an exponential function. For the glucose meal, 52.9\% emptied linearly, and $47 \cdot 1 \%$ exponentially and for the saline meal, $52.6 \%$ emptied linearly, and $47.4 \%$ exponentially. This distribution did not vary significantly between groups of subjects. In view of the variation in the shapes of emptying curves, half time calculated from an exponential function or emptying rate in amount emptied per unit time from the slope of a linear function could not be applied consistently, although, theoretically, an exponential function can describe a very flat curve which approximates to a straight line. Gastric emptying was therefore calculated in several ways. Emptying of the solid meal was expressed as the percentage that remained 100 minutes after the beginning of the meal, and glucose and saline emptying were expressed as the percentage remaining at 30 minutes. The percentages remaining were calculated from the best fit curves by interpolation and by using the decay corrected counts actually obtained at 30 and 100 minutes. Conclusions were identical using either method and the figures given are those obtained using the latter procedure. In addition, half emptying time was calculated from the curve relating log corrected counts to time, and the results obtained using this procedure were the same.

SOLID MEAL

During the initial lag phase (mean 22.95 minutes in controls) the solid portion of the meal was distributed from the fundus to occupy the whole stomach. In one control subject, a double isotope scan was performed using Indium 111-DTPA , 3.7 MBq, to label the liquid phase (orange juice) and Tc99m sulphur colloid, 3.7 $\mathrm{MBq}$ to label the egg, as in the other studies. This confirmed that the liquid phase occupied the whole of the gastric area immediately after consumption while the egg was initially concentrated in the fundus and was gradually 'fed' towards the antrum. There was no significant difference between groups in the length of the lag phase.

The percentage of the solid meal remaining at 100 minutes was significantly greater in patients with anorexia nervosa who were selecting their own diet compared with low weight patients on the refeeding diet, patients with bulimia nervosa and controls $(F(3,41)=4.52, p<0.01)$. Gastric emptying of the solid meal in patients with anorexia nervosa who were receiving the refeeding diet and normal or overweight patients with bulimia nervosa did not differ significantly from controls.

Weight gain among refed anorexic patients had no demonstrable effect on gastric emptying of the solid meal (paired $t$-test). In no control subject was gastric retention at 100 minutes greater than $50 \%$. If delayed gastric emptying is defined as greater than $50 \%$ retention at 100 minutes, it was found in one patient 
Table 3 Gastric emptying results on four patients tested while on a self-selection diet and while receiving the refeeding diet

\begin{tabular}{lllllll}
\hline & \multicolumn{2}{l}{ Solid meal } & \multicolumn{2}{c}{ Glucosemeal } & \multicolumn{2}{c}{ Saline meal } \\
\hline Patient & S-SD & R-FD & S-S-D & R-FD & S-SD & R-FD \\
1 & 51.91 & 17.40 & 98.47 & $81 \cdot 87$ & 29.58 & $49 \cdot 16$ \\
2 & 25.55 & 29.17 & 87.64 & $93 \cdot 18$ & 87.64 & 33.42 \\
3 & 29.83 & 18.06 & 88.51 & $76 \cdot 21$ & $76 \cdot 12$ & 75.13 \\
4 & 69.90 & 23.90 & 100.00 & 77.95 & 13.38 & 40.81 \\
\hline
\end{tabular}

S-S D: self-selection diet. R-F D: refeeding diet. Gastric emptying expressed as per cent of original gastric activity remaining at 100 minutes (solid) or at 30 minutes (liquid meals).

with bulimia nervosa $(10 \%)$, two low weight anorexic patients on the refeeding diet $(16.67 \%)$ and five anorexic patients on the self-selection diet $(50 \%)$. This distribution is significantly different from expected. $\left(\chi^{2}=9 \cdot 14, \mathrm{df}=3, \mathrm{p}<0 \cdot 03\right)$. The delay in gastric emptying among the self-selection diet patients was observed in anorexic patients who suffered bulimic episodes and in patients who did not. Of the five patients in this group whose gastric emptying was delayed, by the above criteria, three suffered from bulimia and two did not. In order to assess the effect of bulimic symptoms on gastric emptying, multivariate analysis of variance was carried out. This confirmed the significant difference between diagnostic groups $(F(3,46)=4 \cdot 21, p=0 \cdot 011)$ but showed no effect of bulimia, vomiting or laxative abuse $(p>0.4)$. The proportion of the variance in solid emptying accounted for by diagnostic group in this analysis was $23.85 \%$, while the combined contribution of the other three variables was $2 \cdot 2 \%$. In four patients, gastric emptying of the solid meal was determined under refeeding diet and self-selection conditions. In the analyses, they are included only in the 'self-selection' group. In two of these, delayed gastric emptying, as defined above, was found, with 51.9 and $69.9 \%$ remaining at 100 minutes. After approximately two weeks of inpatient refeeding, the gastric abnormality reversed (Table 3 ). One out of the eight weight restored patients on the refeeding diet also had delayed emptying. In the 12 patients who received inpatient refeeding, there was no significant correlation (Pearson's $r$ ) between gastric emptying rate of any meal and duration of refeedingthat is, interval between onset of refeeding and initial testing.

\section{GLU COSE EMPTYING}

The emptying of glucose was preceded by a shorter lag phase than that observed for solids (repeated measures analysis of variance: Pillais' test, $p<0.001$, for within-subject differences in lag phase). It did not vary between groups. Analysis of variance showed that the per cent of the meal remaining at 30 minutes was significantly greater for self-selecting anorexic patients compared with controls, patients receiving the refeeding diet, and patients with bulimia nervosa $(F(3,39)=3 \cdot 20, p=0 \cdot 03)$. No other diagnostic group had abnormal glucose emptying and there was no correlation between duration of refeeding and glucose emptying among the 12 refed low weight anorexic patients. The effect of bulimic symptoms was assessed using multivariate analysis of variance. This confirmed the effect of diagnostic group $(F(3,43)=3 \cdot 6, p=0 \cdot 022)$, showed that the presence of bulimia or vomiting did not influence the gastric emptying rate, but revealed that in patients with bulimia nervosa and self-selection diet patients with anorexia nervosa, laxative abuse was associated with reduced glucose emptying rate $(\mathrm{F}(1,43)=5 \cdot 08$, $\mathrm{p}=0.03$ ). Among controls, mean (SD) retention of glucose at 30 minutes was $73.88 \%(11.97)$. Using the control mean +1 SD we classified subjects as having 'slow' ( $\geqslant 86 \%$ retention at 30 minutes) or 'fast' $(<86 \%)$ glucose emptying. 'Slow' emptying was found in two controls $(20 \%)$, one patient with bulimia nervosa $(10 \%)$, one low weight anorexic patient on the refeeding diet $(8 \cdot 3 \%)$, and seven selfselecting anorexic patients $(70 \%) .\left(\chi^{2}=13 \cdot 46, d f=3\right.$, $p<0.004$.) One weight restored patient on the refeeding diet also showed delayed glucose emptying. Of the four patients tested under refeeding and selfselection diet conditions, all had delayed gastric emptying of glucose, as defined above, before treatment (Table 3). After two to three weeks of refeeding, three had improved to under $86 \% 30$ minute retention and one had even slower glucose emptying.

\section{SALINE EMPTYING}

Lag phase and emptying rate showed no significant variation among groups $(p>0.5)$ and were not influenced by the presence of bulimic symptoms. The lag phase for saline was significantly shorter than the solid lag phase (repeated measures analysis of variance, within subject differences in lag phase, Pillais' test, $p<0.001$ ) but not significantly different from the lag phase for glucose.

\section{Discussion}

The present results confirm that delayed gastric emptying is a feature of anorexia nervosa. This study was designed to identify the determinants of delayed gastric emptying in anorexia nervosa: weight loss, acute starvation, and bulimic symptoms were all considered as possible factors contributing to its pathogenesis. It was also considered that delayed emptying might be a specific physiological anomaly in patients with anorexia nervosa, independent of other 
factors, and perhaps predisposing susceptible individuals to the condition. Our data show, however, that while patients who were selecting their own diet had significantly delayed gastric emptying, anorexic patients receiving a refeeding diet did not. Weight gain was not found significantly to influence gastric emptying. It seems likely that the delay in gastric emptying observed in the 'self-selection' patients was associated with their recent dietary intake. The 10 patients in this group reported two patterns of food intake. Six patients engaged in consistent restriction of food intake while the others alternated periods of starvation with episodes of bulimia followed by selfinduced vomiting, laxative abuse or prolonged starvation. Delayed gastric emptying was seen in both of these groups, but not in bulimic patients who were not underweight, indicating that the presence of bulimic symptoms alone does not affect gastric emptying. The results suggest that delayed gastric emptying occurs when there is a reduction of nutrient entering the duodenum sufficient to result in severe weight loss. This reduction may be simply because of inadequate oral intake of food, or alternatively of elimination of food from the stomach by vomiting before it has passed through the pyloric sphincter. It is possible that these changes would be noted in any starving organism, animal or man, and, if this is found to be the case, the regulation of gastric emptying rate would be seen to be determined, in part, by the rate at which food is consumed. This is often, in practice, a function of food supply, implying that postprandial satiety might be enhanced under conditions of famine when prolonged intervals between meals are likely to occur.

The delay in gastric emptying was only observed after ingestion of the solid meal and the glucose solution, showing that the abnormality is not restricted to either solid or liquid meals. Emptying of physiological saline was, however, normal. The solid and glucose meals differ in at least two ways from the saline: they contain caloric nutrient and are both hypertonic. Caloric and osmotic stimuli have been found to delay gastric emptying ${ }^{16} 17$ although the mechanisms by which this inhibition is effected are obscure. McCallum et al ${ }^{4}$ reported that emptying of a hypotonic liquid meal (water) was normal in anorexia nervosa. As water would stimulate duodenal osmoreceptors, the latter study suggests that duodenal feedback by osmotic stimuli is not disturbed in anorexia nervosa. The emptying rates of hypertonic saline and isotonic glucose liquid meals, however, have not been determined, and a disturbance of sensitivity to hypertonic stimuli cannot therefore be excluded.

Inhibition of gastric emptying by nutrients entering the duodenum may involve secretion of gut peptides such as cholecystokinin ${ }^{18}$ and this mechanism might be disturbed in patients with anorexia nervosa. In favour of a disturbance of duodenogastric feedback is our finding, in anorexia nervosa, of a normal lag period for all three types of meal. An abnormal delay in gastric emptying was only observed after some gastric contents had entered the duodenum. On present evidence, a disturbance in duodenal sensitivity to caloric or, perhaps, osmotic, stimuli secondary to severe reduction of food intake seems the most likely mechanism leading to delayed gastric emptying in anorexic patients. The delay in gastric emptying might be mediated by increased pyloric sphincter tone, or decreased gastric contractions. Pyloric activity has not been studied in anorexia nervosa, and a manometric study of gastric contractions in anorexic patients ${ }^{19}$ yielded normal findings. In the latter study, the recent dietary intake of the subjects was not reported and they may not have been tested under the dietary conditions we have found to lead to delayed gastric emptying.

Laxative abuse, among the two groups of patients who were not receiving the inpatient treatment, was found to be associated with significantly slowed emptying of glucose. Purgatives are known to have toxic effects on the autonomic nerves in the intestinal wall ${ }^{20}$ and our results suggest that the stomach may also incur damage as a result of laxative ingestion. Gastric retention in anorexic patients may well exacerbate the characteristic hypophagia, although it did not protect two of our patients (patients 1 and 4 in Table 3) from severe, intractable episodes of bulimia. The degree to which undereating is dependent on enhanced satiety as a result of gastric stasis might be assessed by reversing the abnormality, perhaps pharmacologically, and observing the resulting eating patterns. Although our results suggest that gastric stasis is secondary to undereating, it remains possible that some individuals may develop delayed emptying more readily than others after the onset of dieting, and this sensitivity (perhaps genetically determined) could act as a predisposing factor, allowing common dieting to develop into anorexia nervosa.

A proportion of patients with anorexia nervosa suffer episodes of bulimia followed by self-induced vomiting. The 'efficiency' of this process in reducing calorie absorption presumably depends on the duration of the lag period and the rate of gastric emptying, on the interval between ingestion of the food and self-induced vomiting and on the degree to which the stomach can be emptied of food. Slow gastric emptying might, therefore, contribute to the caloric deficit suffered by bulimic anorexic patients, promote further weight loss, and lead to the particularly resistant form of illness seen in this group of patients. 
One of our subjects (patient 1 in Table 3 ) regularly ate a green apple skin as the first item of her bulimic episode, and was reassured when she saw it return after prolonged vomiting. We have not, however, found evidence of accelerated gastric emptying which might contribute to the pathogenesis of the hyperphagia of bulimia nervosa. A common symptom of patients with anorexia nervosa is slowness of eating and this was observed during the present study. Whether it is related to fear of weight gain, obsessional symptoms, starvation or oesophageal dysfunction ${ }^{5}$ is uncertain, but it did not improve significantly with inpatient treatment, and, surprisingly, it was no less marked among bulimic anorexic patients, when compared with non-bulimic patients.

A rare, but serious, complication of anorexia nervosa, and of malnutrition generally, is acute gastric dilatation. ${ }^{21} 22$ The aetiology of this condition, which has also been described in a patient with bulimia nervosa ${ }^{23}$ is obscure, but it occurs in severely malnourished patients soon after the beginning of inpatient treatment perhaps as a result of rapid refeeding in the presence of delayed gastric emptying. We found that delayed emptying was not observed after a mean of two weeks of inpatient refeeding. While the precise time course of the recovery of delayed gastric emptying during refeeding remains to be determined, acute gastric dilatation seems unlikely to occur as a result of refeeding after two weeks of nutritional treatment.

The authors would like to thank Professor Gerald Russell for permission to study patients under his care and for his helpful comments on the manuscript, the nursing and medical staff on Ward 3 at the Maudsley Hospital and the technical staff in the Departments of Nuclear Medicine and Medical physics at King's College Hospital whose skill and cooperation made this study possible, and $\mathrm{Dr} \mathrm{D}$ Hand for statistical advice. PHR was supported by the Wellcome Trust.

\section{References}

1 Dubois A, Gross HA, Ebert MH, Castell DO. Altered gastric emptying and secretion in primary anorexia nervosa. Gastroenterology 1979; 77: 319-23.

2 Holt S, Ford MJ, Grant S, Heading RC. Abnormal gastric emptying in primary anorexia nervosa. $\mathrm{Br} J$ Psychiatry 1981; 139: 550-2.

3 Russell DMcR, Freedman ML, Feiglin DHI, Jeejeebhoy KN, Swinson RP, Garfinkel PE. Delayed gastric emptying and improvement with domperidone in a patient with anorexia nervosa. Am J Psychiatry 1983; 140: $1235-6$.
4 McCallum RW, Grill BB, Lange R, Planky M, Glass EE. Definition of a gastric emptying abnormality in patients with anorexia nervosa. Dig Dis Sci 1985; 30: 713-22.

5 Stacher G, Kiss A, Wiesnagrotzki S, Bergmann H, Hobart J, Schneider C. Oesophageal and gastric motility disorders in patients categorised as having primary anorexia nervosa. Gut 1986; 27: 1120-6.

6 Russell GFM. Bulimia nervosa: an ominous variant of anorexia nervosa. Psychol Med 1979; 9: 429-48.

7 Wright RA, Krinsky S, Fleeman C, Trujillo J, Teague E. Gastric emptying and obesity. Gastroenterology 1983; 84: 747-51.

8 Russell GFM. Anorexia nervosa and bulimia nervosa. In: Russell GFM, Hersov LA, eds. Handbook of psychiatry 4. Neuroses and personality disorders. Cambridge: Cambridge University Press, 1983: 285-98.

9 Metropolitan Life. Statistical bulletin of the Metropolitan Life Insurance Company. 1983; 64.

10 Garner DM, Garfinkel PE. The eating attitudes test: an index of the symptoms of anorexia nervosa. Psychol Med 1979; 9: 273-9.

11 Van Strien T, Frijters J, Bergers G, Defares P. The Dutch eating behaviour questionnaire (DEBQ) for assessment of restrained, emotional and external eating behaviour. Int J Eat Dis 1985; 5: 295-315.

12 Brener W, Hendrix TR, McHugh PR. Regulation of the gastric emptying of glucose. Gastroenterology 1983; 85: 76-82.

13 Minami H, McCallum RW. The physiology and pathophysiology of gastric emptying in humans. Gastroenterology 1984; 86: 1592-610.

14 Christian PE, Moore JG, Sorenson JA. Effects of meal size and correction technique on gastric emptying time; studies with two tracers and opposed detectors. J Nucl Med 1980; 21: 883-5.

15 Moore JG, Christian PE, Coleman RE. Gastric emptying of varying meal weight and composition in man. Evaluation by dual liquid- and solid-phase isotopic method. Dig Dis Sci 1981; 26: 16-22.

16 Hunt JN. Some properties of an alimentary osmoreceptor mechanism. J Physiol 1956; 132: 267-88.

17 Hunt JN, Stubbs DF. The volume and energy content of meals as determinants of gastric emptying. J Physiol 1975; 245: 209-25.

18 Moran TH, McHugh PR. Cholecystokinin suppresses food intake by inhibiting gastric emptying. Am J Physiol. 1982; 242 (Regulatory integrative comp. physiol. 11): R491-R497.

19 Silverstone JT, Russell GFM. Gastric 'hunger' contractions in anorexia nervosa. Br J Psychiatry 1967; 113: 257-63.

20 Smith B. Effect of irritant purges on the myenteric plexus in man and the mouse. Gut 1968; 9: 139-43.

21 Russell GFM. Acute dilatation of the stomach in a patient with anorexia nervosa. BrJ Psychiatry 1966; 112: 203-7.

22 Markowski B. Acute dilatation of the stomach. Br Med J 1947; 2: 128-30.

23 Mitchell JE, Pyle RL, Miner RA. Gastric dilatation as a complication of bulimia. Psychosomatics 1982; 23: 96-7. 